\title{
Effects of a Single Pill 3-drug Combination of Lamivudine, Nevirapine and Zidovudine on Blood Parameters and Liver Histology in Female Wistar Rats
}

\author{
Adesanya Olamide Adewale ${ }^{1, *}$, Otulana Olatoye Jubril ${ }^{1}$, Huthman Ibrahim Oluwaseun ${ }^{1}$, \\ Huthman Adebukola Selimot ${ }^{2}$, Adesanya Rotimi Ayoola ${ }^{3}$ \\ ${ }^{1}$ Department Of Anatomy, Faculty of Basic Medical Science, Olabisi Onabanjo University Ogun State, Nigeria \\ ${ }^{2}$ Federal Institute Of Industrial Research Oshodi (FIIRO) \\ ${ }^{3}$ Department of Medical Pharmacology \& Therapeutics, Faculty of Basic Medical Sciences, Obafemi Awolowo University, Ile-Ife, Nigeria
}

\begin{abstract}
Due to the stigma associated with HIV infection, HIV infected persons in Nigeria, have been found to remove the packs of their drugs and transferring the contents into non antiretroviral drug containers especially multivitamin containers, as a result unwary individuals have stumbled on such drugs and accidentally consumed it eventually leading to a host of complains such as dizziness, vague dreams, cramps etc. We therefore decided to study the effects of a single pill 3-drug combination of Lamivudine, Nevirapine and Zidovudine on the liver histology and blood parameters in the adult female rats. The rats were divided into two groups of 5 animals per group: one group received drug at a dose of $1.85 \mathrm{mg} / 100 \mathrm{~g}$ BW dissolved in normal saline while the control group received normal saline $0.5 \mathrm{ml} / 100 \mathrm{~g}$ BW for three weeks. From our observation we discovered that there was an aberration in liver histology of the experimental group in that the central vein was dilated as compared to the control group and also discovered that the drug had some effect on the blood parameters, which include among other things a significant decrease in PCV and a significant increase in WBC. The enlargement of the central vein of the liver observed in this study may be due to the presence of Nevirapine in the drug regimen and this may be of serious implication on liver function.
\end{abstract}

Keywords Lamivudine, Nevaripine and Zidovudine, Stigma, Liver Histology

\section{Introduction}

Acquired immune deficiency syndrome or acquired immunodeficiency syndrome (AIDS) is a disease of the human immune system caused by the human immunodeficiency virus $(\mathrm{HIV}) \mathrm{.}^{[1][2][3]}$ This condition progressively reduces the effectiveness of the immune system and leaves individuals susceptible to opportunistic infections and tumors. HIV is transmitted through direct contact of a mucous membrane or the bloodstream with a bodily fluid containing HIV, such as blood, semen, vaginal fluid, pre-seminal fluid, and breast milk. $^{[4][5]}$.

AIDS is now a pandemic. ${ }^{[6]}$ In 2007 , it was estimated that 33.2 million people lived with the disease worldwide, and that AIDS killed an estimated 2.1 million people, including 330,000 children. ${ }^{[7]}$ Over three-quarters of these deaths occurred in sub-Saharan Africa. ${ }^{[7]}$ Genetic research indicates that HIV originated in west-central Africa during the late nineteenth or early twentieth century. ${ }^{[8][9]}$ AIDS was first

* Corresponding author:

lammyworth@yahoo.com (Adesanya Olamide Adewale)

Published online at http://journal.sapub.org/ajmms

Copyright (C) 2012 Scientific \& Academic Publishing. All Rights Reserved recognized by the U.S. Centers for Disease Control and Prevention in 1981 and its cause, HIV, identified in the early 1980s. ${ }^{[10]}$ Although treatments for AIDS and HIV can slow the course of the disease, there is currently no known cure or vaccine. Antiretroviral treatment reduces both the mortality and the morbidity of HIV infection, but these drugs are expensive and routine access to antiretroviral medication is not available in all countries. ${ }^{[11]}$

Reverse transciptase inhibitors (Lamivudine (3TC), nevirapine (NVP), zidovudine (AZT)) are drugs that inhibit both types (1 and 2) of HIV reverse transcriptase and also the reverse transcriptase of hepatitis B. Lamivudine (3TC) a synthetic antiretroviral agent is a dideoxynucleoside reverse transciptase inhibitor. 3TC is a negative enantiomer of a dideoxy analog of cytidine and is structurally similar to zalcitabine. Following conversion to a pharmacologically active metabolite, 3TC apparently inhibts replication of human retroviruses by interferening with viral RNA- directed DNA polymerase (reverse transcriptase). 3TC therefore exerts a virustatic effect against retroviruses by acting as a reverse transciptase inhibitor ${ }^{[12]}$.

Zidovudine (AZT) is an antiviral agent which is a potent inhibitor of replication of $\mathrm{HIV}^{[13]}$. AZT is phosphorylated in both infected and uninfected cells to the monophosphate 
(MP) derivative by cellular thymidine kinase. Subsequently phosporylated of AZT-MP to the diphosphate (DP), then the triphosphate (TP) derivative is catalyzed by cellular thymidylate kinases respectively. AZT -TP acts as an inhibitor of and substrate for the viral reverse transcriptase. The formation of further proviral DNA is blocked by incorporation -MP into the chain and subsequently chain termination. Competition by AZT-TP for HIV reverse transcriptase is approximately 100 -fold greater than for cellular DNA polymerase alpha ${ }^{[14]}$.

Nevirapine (NVP) acts by reversibly inhibiting the activity of HIV-1 reverse transciptase, an enzyme which directs the polymerization of DNA from viral RNA, a necessary component for HIV -1 replication. For HIV-1 as well as other retroviruses, the viral polymerase has a binding site for nucleoside triphosphate, a site for binding the RNA template primer, and catalytic sites for both the polymerase and ribonuclease $\mathrm{H}$ reactions. The inhibition of reverse transcriptase directed polymerization of DNA from viral RNA has been an important therapeutic target for the treatment of HIV-1 infection, which was initially reported with the nucleoside analogue AZT. Unlike the nucleoside analogues, NVP binds directly to the reverse transcriptase at amino acid residues 181 and 188 . This site is close to, but not directly at the polymerase catalytic site on the large subunit of the heterodimeric reverse transcriptase. The binding of NVP to reverse transcriptase occurs primarily through hydrophobic interactions to a pocket formed by seven strands, nevaripine appears to block reverse transcriptase polymerase by altering the position of critical amino acids within the enzymatic site. As a result, the rate of the chemical reaction catalyzed by the reverse transciptase is significantly slowed. NVP does not exhibit activity against other viral polymerase, including HIV-2 and simian immunodeficiency virus reverse transcriptase. NVP has been studied in several combination regimens for the treatment of HIV ${ }^{[15]}$

AIDS stigma exists around the world in a variety of ways, including ostracism, rejection, discrimination, avoidance of HIV infected people, compulsory HIV testing without prior consent or protection of confidentiality, violence against HIV infected individuals or people who are perceived to be infected with HIV and the quarantine of HIV infected individuals. ${ }^{[16]}$ Stigma-related violence or the fear of violence prevents many people from seeking HIV testing, returning for their results, or securing treatment, possibly turning what could be a manageable chronic illness into a death sentence and perpetuating the spread of HIV.${ }^{[16]}$ Often, AIDS stigma is expressed in conjunction with one or more other stigmas, particularly those associated with homosexuality, bisexuality, promiscuity, prostitution, and intravenous drug use. In many developed countries, there is an association between AIDS and homosexuality or bisexuality, and this association is correlated with higher levels of sexual prejudice such as anti-homosexual attitudes. ${ }^{[17]}$ There is also a perceived association between AIDS and all male-male sexual behaviour, including sex between uninfected men. ${ }^{[18]}$
Due to the problem of stigma associated with HIV infection, HIV infected persons in Nigeria, have been found to remove packs of their drugs and transferring the contents into non antiretroviral drug containers especially multivitamin containers, as a result unwary individuals have stumbled on such drugs and accidentally consumed it eventually leading to a host of complains such as vague dreams, cramps etc after consumption. We therefore decided to study the effects of these drugs on the morphology of the liver and blood parameter in the adult female Wistar rats.

\section{Materials and Methods}

\subsection{Animals}

10 Adult female Wistar rats weighing between $150-250 \mathrm{~g}$ were obtained from the colony breed of the animal house of the Department of Anatomy, Obafemi Awolowo University, Ile-Ife, Nigeria. They were housed in individual cages in a room with ambient temperature. The rats were fed with standard pellet diet (Sesco Feeds Ikenne, Nigeria) and given water ad-libitum. The weights of the rats were taken daily.

\subsection{Study Group/ Drug Administration}

The animals were divided into 2 groups as follows Group $\mathrm{A}(\mathrm{n}=5)$ : Rats received daily administration of $1.85 \mathrm{mg} / 100 \mathrm{~g}$ BW of anti-retroviral therapy (ART) drugs i.e. single pill 3-drug combination of Lamivudine, Nevirapine and $\mathrm{Zi}$ dovudine for three weeks and Group B ( $\mathrm{n}=5)$ : Control group received daily administration of $0.5 \mathrm{ml}$ of normal saline. The Animals were induced once daily for three weeks. The drug was manufactured by Aurobindo Pharma Limited, unit III, Survey No. 313, Bachupally Village, Quthubullapur Mandal, Ranga Reddy District (A.P), India.

\subsection{Histology}

The animals were sacrificed and the abdominal cavity of each rat opened, the liver taken out. The organ was fixed in $10 \%$ formol-saline. After complete fixation the blocks was embedded in paraffin and sections cut at $5 \mu \mathrm{m}$ (micron) which was then stained with haematoxylin and eosin and mounted in Canada balsam. Microscopic examination of the sections was then carried out under a light microscope.

\subsection{Blood Parameters}

A full blood count showing the haematological parameters was then done.

\subsection{Statistical Analysis}

Statistical analysis was done using the student-t package and values in the tables were represented as mean \pm standard deviation. Values of significant were taken at $\mathrm{P}<0.05$.

\section{Results}

The result of the experiment shows an enlargement of the central vein of the liver in the experimental group as com- 
pared to the control group when viewed under a light microscope. The result also showed an increase in the monocyte, neutrophil and WBC as compared to the experimental group although mild increases were also observed eosinophil count. On the other hand a slight decrease in the lymphocyte and PCV level was also observed in the experimental group as compared to the control. The result further showed that the drug regimen did not have any effect on the experimental groups' body weight as compared to the control.

\section{LIVER HISTOLOGY - CONTROL GROUP}
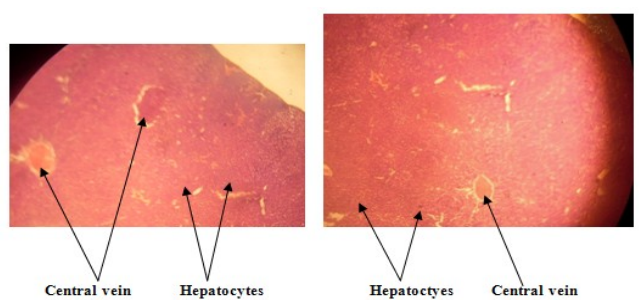

Figure 1. Liver histo-architecture of the control group that was given normal saline showed that the central vein appears normal and the hepatocytes intact

\section{EXPERIMENTAL GROUP}
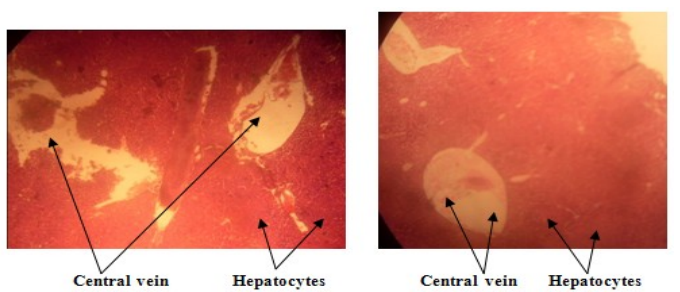

Figure 2. The effect of a single pill 3-drug combination of Lamivudine, Nevirapine and Zidovudine on the liver histo-architecture of the experimental group shows dilation of the central vein

\section{BLOOD PARAMETERS}

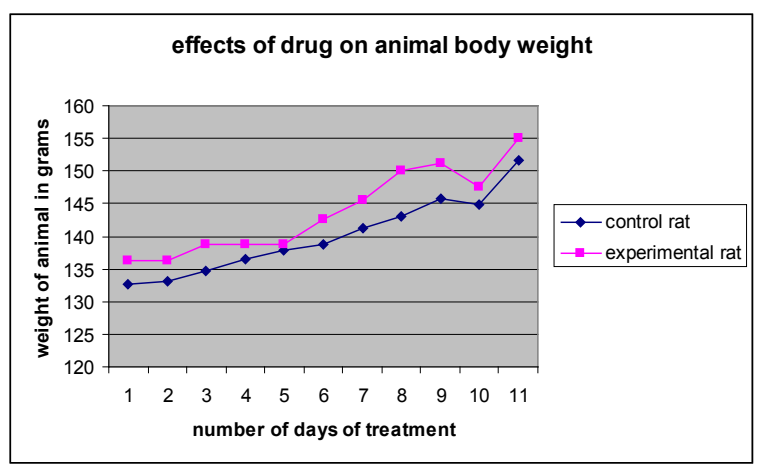

Figure 3. Shows that the drug regimen did not have any effect on the experimental group's body weight as compared to be control

\section{OTHER FINDINGS}

The treated animal developed rashes on the snout one week into the study and also displays some level of sluggishness few hours after the treatment, suggesting neurological impairment.

\section{Discussion}

The liver is prone to xenobiotic-induced injury because of its central role in xenobiotic metabolism and its portal location within the circulatory system ${ }^{[19]}$ ARV is not an exemption and its constituents will be acted upon, by the cells of the liver. Normal doses approved for human subject was worked out and administered to represent human exposure of AZT, NVP, and 3TC combination. It could be observed from the result obtained in this study that normal dose of AZT, NVP and 3TC combination caused an abnormal morphology of the liver in which there was dilatation of the central vein of the liver. This may be due to the NVP present in the ARV which has been reported to be hepatotoxic ${ }^{[20]}$ and also agrees with the finding of Pao et al, who reported development of fatty liver with mild portal inflammation following the administration of stavudine and abacavir in HIV infected patients. ${ }^{[20]}$ There was an increment in the percentage of white blood cells caused by ARV administration, this may be due to the inflammatory reaction of some of the animal's organs and tissues to the ARV drug as compared to the control when administered daily for 3 weeks. ${ }^{[21]}$ There was development of rashes on the snout of the animal one week into the study, these may be some of the tissue reaction to the highly toxic but potent ARV and also observed were some form of sluggishness among the animals.

There was also a slight decrease in the number of lymphocyte and an increased number of monocyte which is in support of the report of Gibson J. ${ }^{[22]}$ which claims that number of lymphocyte decrease in viral infection while monocyte increase where there is a chronic infection. In fact, the administration of ARV in this study caused a reaction in the animal which mimics a form of viral and chronic infection. The drug also caused a reduction of the PCV which implies that the drug suppresses hematopoiesis in the bone marrow, this is agrees with a report by Gilks $\mathrm{C}$ and Vercauteren $\mathrm{G}$ that states the hematoxic ffect of AZT on bone marrow one of the component of this ARV drug ${ }^{[23]}$

Table 1. The effect of a single pill 3-drug combination of Lamivudine, Nevirapine and Zidovudine on blood parameter in rats

\begin{tabular}{|c|c|c|}
\hline & CONTROL & $\begin{array}{c}\text { EXPERI- } \\
\text { MENTAL }\end{array}$ \\
\hline PCV (\%) & $44.6 \pm 2.70$ & $44.4 \pm 1.82 *$ \\
\hline $\begin{array}{c}\text { WBC (Per } \\
\text { Microlitre) }\end{array}$ & $4400 \pm 230.22$ & $4880 \pm 313.1 *$ \\
\hline $\begin{array}{c}\text { NEUTRO- } \\
\text { PHIL (\%) }\end{array}$ & $16.4 \pm 1.42$ & $17.2 \pm 0.85 *$ \\
\hline $\begin{array}{c}\text { LYMPHO- } \\
\text { CYTE (\%) }\end{array}$ & $80.6 \pm 1.5$ & $79.8 \pm 1.92 *$ \\
\hline $\begin{array}{c}\text { EOSINOPHIL } \\
(\%)\end{array}$ & $1 \pm 0$ & $1.2 \pm 0.45 *$ \\
\hline $\begin{array}{c}\text { MONOCYTE } \\
(\%)\end{array}$ & $1 \pm 0$ & $2.6 \pm 0.89 *$ \\
\hline
\end{tabular}

Values were expressed as mean \pm standard deviation $* p<0.05$

\section{Conclusions}

It can be concluded from the result of this work that a single pill 3-drug combination of Lamivudine, Nevirapine and Zidovudine led to abnormal morphology of the liver in 

and Zidovudine on Blood Parameters and Liver Histology in Female Wistar Rats

female adult Wistar rats. It also caused increase in WBC and monocyte count as when compared to the control, therefore, one can advice that this drug should not be use except on doctor's advice as the negative effect may be of great consequence if used without medical guide. Care must be taken when given out this drug to patients who want to use them as pre/post exposure prophylaxis. It is recommended that further work should be done to elucidate the effects of Non Reverse Transcriptase Inhibitor (3TC, NVP \& AZT) combination on liver histology and blood parameters in female Wistar Rats.

\section{REFERENCES}

[1] Sepkowitz KA (2001). AIDS--the first 20 years: N. Engl. Journal; 344 (23): 1764-72. z

[2] Weiss RA (1993). How does HIV cause AIDS?;Science journal; 260 (5112): 1273-9.

[3] Cecil, Russell (1988). Textbook of Medicine. Philadelphia: Saunders; pp. 1523, 1799.

[4] Divisions of HIV/AIDS Prevention (2003). "HIV and Its Transmission". Centers for Disease Control \& Prevention.

[5] San Francisco AIDS Foundation (2006). "How HIV is spread".

[6] Kallings LO (2008). The first postmodern pandemic: 25 years of HIV/AIDS: J Intern Med; 263 (3): 218-43.

[7] UNAIDS, WHO. "2007 AIDS epidemic update" 2007.

[8] Gao F, Bailes E, Robertson DL (1999). Origin of HIV-1 in the Chimpanzee Pan troglodytes troglodytes: Nature; 397 (6718): 436-441.

[9] Worobey M, Gemmel M, Teuwen DE (2008). Direct evidence of extensive diversity of HIV-1 in Kinshasa by 1960 : Nature; 455 (7213): 661-4.

[10] Gallo RC (2006). A reflection on HIV/AIDS research after 25 years. Retrovirology; 3: 72.

[11] Palella FJ Jr, Delaney KM, Moorman AC (1998). Declining morbidity and mortality among patients with advanced human immunodeficiency virus infection. HIV Outpatient Study Investigators: N. Engl. J. Med; 338 (13): 853-860.
[12] Fox Z, Dragsted UB, Gerstoft J (2006). A randomized trial to evaluate continuation versus discontinuation of lamivudine in individuals failing a lamivudine-containing regimen: The COLATE trial: Antiviral Therapy; 11 (6): 761-70.

[13] Antiretroviral Pregnancy Registry Steering committee. Antiretroviral pregnancy Registry International Interim report for 1st January 1989- 31st July 2008. Wilmington NC registration coordinating center; 2008

[14] Second Meeting of the Subcommittee of the Expert Committee on the Selection and Use of Essential Medicines (29 september-3rd October 2008).

[15] Tatfeng youtchou Mirabeau, Nwobu Gilbert Obinna, Okodua marcellinus, Agbolanhor Dennis Edo, Agba Mattiahs Ikenna, Agwu Ezera.( 2005). Effect of Lamivudine (Epivir), Nevirapine (Vivumine) and stavudine (Starvir) on CD4+ counts on Hiv patients Attending University of Benin teaching Hospital (UBTH), Benin, Edo State, Nigeria: Kuwait Medical Journal; $37(2): 86-90$

[16] Ogden J, Nyblade L. (2005). Common at its core: HIV-related stigma across contexts (PDF): International Center for Research on Women

[17] Herek GM, Capitanio JP, Widaman KF (2002). HIV-related stigma and knowledge in the United States-prevalence and trends, 1991-1999: Am J Public Health; 92 (3): 371-7.

[18] Herek GM, Capitanio JP (1999). AIDS Stigma and sexual prejudice: American Behavioral Scientist; 42 (7): 1130-1147.

[19] Jones AL (1996). Anatomy of the normal liver. In: Zakin D, Boyer TD, Eds. Hepatology: a textbook of liver disease, 3rd ed. Philadelphia: WB Saunders,; 3-32.

[20] D Pao, C Watson, B Peters, S B Lucas, R F Miller (2001). Hyperlactataemia and hepatic steatosis- mitochondrial toxicity of nucleoside reverse transcriptase inhibitors: Sex Transm Inf; 77: 381-384.

[21] Parth S Mehta (2004). HIV curriculum for the health professional: Hematological manifestation of HIV/AIDS; Pg 222228 .

[22] Gibson Joel (1993). Step perspective: publication of education project, STEP; vol 5, number1. A

[23] Gilks, C and Vercauteren G (2004): WHO consultation on technical and operational recommendations for scale-up of laboratory services and monitoring HIV antiretroviral therapy in resource-limited settings. 